\title{
Increasing Provasculature Complexity in the Arabidopsis Embryo May Increase Total Iron Content in Seeds: A Hypothesis
}

\author{
Hannetz Roschzttardtz*, Sofía Bustos, Maria F. Coronas, Miguel A. Ibeas, \\ Susana Grant-Grant and Joaquín Vargas-Pérez
}

Departamento de Genética Molecular y Microbiología, Facultad de Ciencias Biológicas, Pontificia Universidad Católica de Chile, Santiago, Chile

\section{OPEN ACCESS}

Edited by:

Felipe Klein Ricachenevsky, Universidade Federal de Santa Maria,

Brazil

Reviewed by:

Maria Noelle Lo,

University of Colorado Boulder,

United States

Diego Fabian Gomez-Casati, National University of Rosario,

CONICET, Argentina

*Correspondence:

Hannetz Roschzttardtz hroschzttardtz@bio.puc.cl

Specialty section:

This article was submitted to Plant Nutrition,

a section of the journal

Frontiers in Plant Science

Received: 19 December 2016

Accepted: 22 May 2017

Published: 08 June 2017

Citation:

Roschzttardtz H, Bustos S, Coronas MF, Ibeas MA,

Grant-Grant S and Vargas-Pérez $J$ (2017) Increasing Provasculature Complexity in the Arabidopsis Embryo May Increase Total Iron Content in Seeds: A Hypothesis. Front. Plant Sci. 8:960 doi: 10.3389/fpls.2017.00960
Anemia due to iron deficiency is a worldwide issue, affecting mainly children and women. Seed iron is a major source of this micronutrient for feeding, however, in most crops these levels are too low to meet daily needs. Thus, increasing iron allocation and its storage in seeds can represent an important step to enhance iron provision for humans and animals. Our knowledge on seed iron homeostasis is mainly based on studies performed in the model plant Arabidopsis thaliana, where iron accumulates in endodermis cells surrounding the embryo provasculature. It has been reported that cotyledon provasculature pattern complexity can be modified, thus we hypothesize that changes in the complexity of embryo vein patterns may affect total iron content in Arabidopsis seeds. This approach could be used as basis to develop strategies aimed to biofortify seeds.

Keywords: iron, embryo, endodermis, provasculature, biofortification

\section{INTRODUCTION}

Malnutrition is one of the greatest health problems in the world, causing chronic diseases, altered physical and mental development, as well as reduced socioeconomic development of the countries most affected. The major problem of micronutrient deficiency worldwide is iron deficiency, which is a serious public health problem and a major concern for the World Health Organization (WHO). According to WHO, $30 \%$ of the world's population suffers anemia, affecting mainly women and children. Also, the role of iron in fertility and seed yield is an important agronomical trait, because low iron bioavailability in soils, widespread in arable soils, limits plant fertility (Guerinot and Yi, 1994). Recent evidence shows that the amount of micronutrients in seeds has declined systematically since the beginning of the so-called green revolution in the 1960s (Fan et al., 2008; DeFries et al., 2015). Crops biofortification is an agronomic tool that can be implemented to solve malnutrition by increasing the natural content of micronutrients in plants. Biofortification can improve the nutritional content of staple foods that are consumed by the population, resulting in a cost-efficient and sustainable way of delivering nutrients to poor or rural populations with limited access to markets or health centers that provide fortified foods or supplements. To modify the iron content of seeds for human consumption (biofortification) is an attractive alternative to combat iron deficiency, however, mineral loading of seeds is strictly controlled in plants and for instance an increase in root absorption does not produce necessarily seeds with higher 


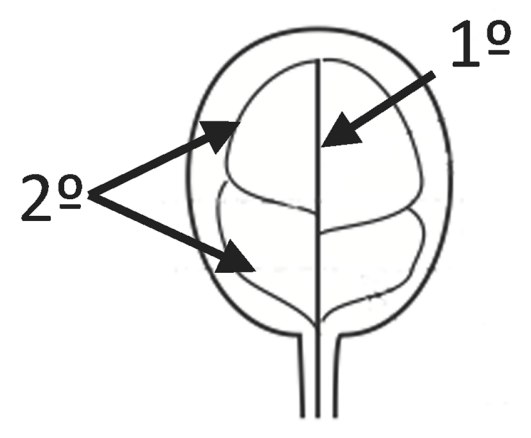

FIGURE 1 | Provasculature patterns in Arabidopsis cotyledons. In the figure $1^{\circ}$ indicates the primary vein and $2^{\circ}$ secondary veins. $1^{\circ}$ and $2^{\circ}$ are forming 4 areoles in this case.

mineral content (Waters and Grusak, 2008; Murgia et al., 2012). Environmental factors and transport activity may be also limiting seed Fe content. Recently, a mechanism depending on ascorbate efflux in plant embryos has been described and it could play a role in the control of iron loading in seeds (Grillet et al., 2014). However, still little is known about transport activity and iron content in seeds. In addition to metabolic and physiological approaches, plant anatomy need to be tackled in order to reach micronutrient improvement in plants (Vasconcelos et al., 2016).

\section{IRON DISTRIBUTION IN Arabidopsis EMBRYO}

Elemental imaging of metals using Arabidopsis seeds were previously obtained by X-ray tomography fluorescence (XRF) and Perls/DAB staining: iron accumulates in the vacuoles of the endodermis/perivascular cell layer during Arabidopsis thaliana seed maturation (Kim et al., 2006; Roschzttardtz et al., 2009; Mary et al., 2015). Endodermis cells surround provasculature and iron can be used as a marker of provasculature patterning in Arabidopsis embryos (Roschzttardtz et al., 2010, 2014). By the time seeds reach maturity the embryo has developed a complete array of procambial strands. After germination, the embryonic provasculature strands differentiate into vascular bundles consisting mostly of phloem and xylem (Sieburth and



FIGURE 3 | Hypothetic model indicating that Fe total content in seeds will increase in a population of embryos with maximal provasculature complexity. WT data is from Roschzttardtz et al. (2014). HypMut correspond to a hypothetical seed population containing cotyledons with higher provasculature complexity.

Deyholos, 2006). In Arabidopsis, the vein pattern in embryo cotyledons is simple, consisting in primary and secondary veins that can be connected forming areoles (Figure 1). Embryo provasculature cotyledon pattern categories can be defined using Perls/DAB staining, and different degrees of pattern complexity can be found in cotyledons from wild type seeds (Figures 2A,B). Simplest complexity pattern can have only two areoles while four and more areoles are found in the cases of higher complexity (Figures 2A,B). Interestingly, in a wild type seed population, cotyledons with different provasculature complexity patterns are found (Figures $\mathbf{2 A}, \mathbf{3}$ ). These categories also can be defined in cotyledons by visualizing lignin autofluorescence from mature xylem cells in 1-week-old seedlings (Cnops et al., 2006; Truernit et al., 2012; Roschzttardtz et al., 2014). Different reports indicate that provasculature vein pattern can be modified either decreasing or increasing its complexity (Carland et al., 2002, 2016; Sieburth et al., 2006; Truernit et al., 2012; Roschzttardtz et al., 2014). Noteworthy, a change in provasculature complexity does not lead to changes in cotyledon size (Figure 2B). Considering that cotyledon veins are surrounded by the endodermis, does an increase in the

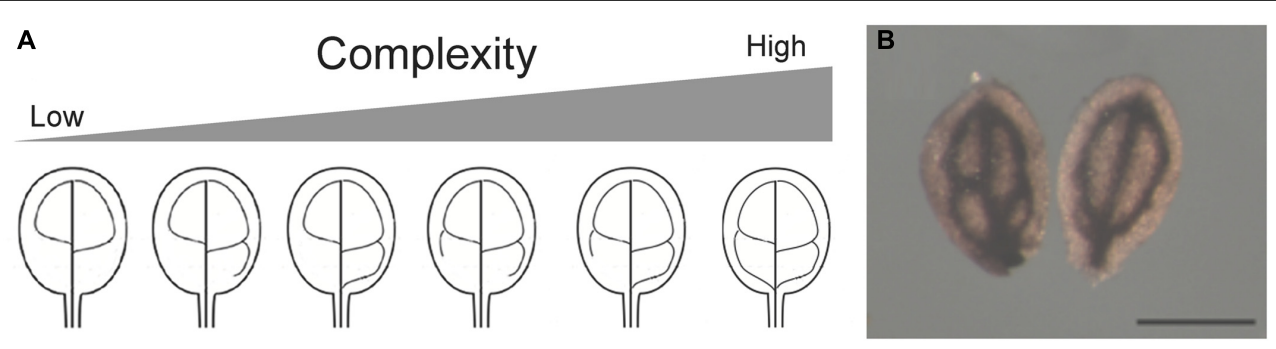

FIGURE 2 | (A) Arabidopsis cotyledon provasculature complexity. Different provasculature patterns found in wild type embryos are shown. (B) Cotyledons with different provasculature complexities have the same size. Mature wild-type cotyledons were stained with Perls/DAB to detect iron accumulation around the provasculature. Bar, $50 \mu \mathrm{m}$. 
TABLE 1 | Estimation of iron increase in cotyledons with high provasculature complexity.

\begin{tabular}{|c|c|c|c|c|c|}
\hline Vein Complexity (a) & $\begin{array}{l}\text { Length Ratio of } \\
\text { Provasculature }\end{array}$ & $\begin{array}{l}\text { Distribution in WT } \\
\text { (c) }\end{array}$ & $\begin{array}{l}\text { Distribution in } \\
\text { HypMut (d) }\end{array}$ & $\begin{array}{c}\text { Iron by } \\
\text { Complexity WT }\end{array}$ & $\begin{array}{c}\text { Iron by Complexity } \\
\text { HypMut }\end{array}$ \\
\hline & 0.7 & 4.2 & 0.0 & 3.5 & 0.0 \\
\hline & 0.8 & 16.7 & 0.0 & 15.9 & 0.0 \\
\hline & 0.8 & 43.1 & 0.0 & 45.6 & 0.0 \\
\hline & & & $\begin{array}{l}\text { Total Relative Iron } \\
\text { Content }(\mathrm{e})\end{array}$ & 100 & 126.5 \\
\hline
\end{tabular}

(a) Vein provasculature complexity defined according to Roschzttardtz et al. (2014). Category "others" was not included.

(b) Length ratio of provasculature in cotyledons of 4-1's category.

(c) Percentage of cotyledons according to Roschzttardtz et al. (2014).

(d) Percentage of cotyledons according to Figure 3.

(e) Only cotyledons.

complexity of the embryo provasculature pattern lead to an increase in Fe content in the seed? In other words, we hypothesize that as consequence of increasing the proportion of cotyledons with four areoles or more, the volume of provasculature and endodermis will also be increased, and therefore iron content will be higher (Figure 3). Considering that overaccumulation of free Fe might lead to toxicity by production of reactive oxygen species (Ravet and Pilon, 2013), increasing iron content in seeds through the increase of the number of endodermal cells where iron accumulates in vacuoles can prevent oxidative stress. The mechanisms that control provasculature pattern complexity in Arabidopsis are not well defined, however, it has been described that OPS or VCC overexpressing plants produce seeds with increased vein pattern complexity in cotyledons (Truernit et al., 2012; Roschzttardtz et al., 2014). Overexpression of OPS leads to premature phloem differentiation, suggesting that phloem development could positively affect vascular patterning (Truernit et al., 2012). One factor involved in vascular patterning is auxin (Scarpella et al., 2010). However, in VCC overexpressing plants, changes in the provascular pattern complexity seem to be not related to auxin transport (Roschzttardtz et al., 2014).

In order to address our hypothesis, analysis of Fe content and distribution in different genotypes will be relevant. For instance, seeds from VCC and OPS overexpressing plants showed an increase of provasculature pattern complexity (Truernit et al., 2012; Roschzttardtz et al., 2014) and should be an excellent material to be used as proof of concept. In an opposite way, a decrease in provasculature complexity should lead to the production of seeds with less Fe content. If our hypothesis is correct, seeds from vcc ops double mutant plants that have a less complex provasculature pattern in cotyledons (Roschzttardtz et al., 2014), should have a significant decrease in total Fe content. An estimation of the possible iron increasing in cotyledon is shown in Table 1. In the case of a distribution of vein complexity like proposed for a hypothetical mutant shown in Figure 3, the total iron content could increase around to $26 \%$ in cotyledons.

Elucidating the genetic control and signaling involved in the provasculature pattern complexity could be an important step in order to develop strategies to increased iron content in seeds.

\section{CONCLUDING REMARKS}

In the Arabidopsis embryo, iron accumulates in the endodermis that surrounds the provasculature. We hypothesize that as an indirect consequence of increasing of provasculature pattern complexity, Fe content in seeds will also increase. This could be used as concept to develop strategies aimed to biofortify seeds.

\section{AUTHOR CONTRIBUTIONS}

All authors listed have made a substantial, direct and intellectual contribution to the work, and approved it for publication.

\section{FUNDING}

This work was funded by FONDECYT 1160334 (Chilean Government) and INTER 6809 (Pontificia Universidad 
Católica de Chile-VRI) to HR, and Millennium Nucleus Center for Plant Systems and Synthetic Biology (NC130030). Ph.D. students work was supported by Conicyt-Chile grants 21160350 (to MI), 21170951 (to SG) and 21151344 (to JV-P).

\section{REFERENCES}

Carland, F., Defries, A., Cutler, S., and Nelson, T. (2016). Novel vein patterns in Arabidopsis induced by small molecules. Plant Physiol. 170, 338-353. doi: 10.1104/pp.15.01540

Carland, F., Fujioka, S., Takatsuto, S., Yoshida, S., and Nelson, T. (2002). The identification of CVP1 reveals a role for sterols in vascular patterning. Plant Cell 14, 2045-2058. doi: 10.1105/tpc.003939

Cnops, G., Neyt, P., Raes, J., Petrarulo, M., Nelissen, H., Malenica, N., et al. (2006). The TORNADO1 and TORNADO2 genes function in several patterning processes during early leaf development in Arabidopsis thaliana. Plant Cell 18, 852-866. doi: 10.1105/tpc. 105.040568

DeFries, R., Fanzo, J., Remans, R., Palm, C., Wood, S., and Anderman, T. L. (2015). Metrics for land-scarce agriculture. Science 349, 238-240. doi: 10.1126/science. aaa5766

Fan, M., Zhao, F., Fairweather-Tait, S., Poulton, P., Dunham, S., and McGrath, S. (2008). Evidence of decreasing mineral density in wheat grain over the last 160 years. J. Trace Elem. Med. Biol. 22, 315-324. doi: 10.1016/j.jtemb.2008. 07.002

Grillet, L., Ouerdane, L., Flis, P., Hoang, M., Isaure, M., Lobinski, R., et al. (2014). Ascorbate efflux as a new strategy for iron reduction and transport in plants. J. Biol. Chem. 289, 2515-2525. doi: 10.1074/jbc.M113.514828

Guerinot, M., and Yi, Y. (1994). Iron: nutritious, noxious, and not readily available. Plant Physiol. 104, 815-820. doi: 10.1104/pp.104.3.815

Kim, S., Punshon, T., Lanzirotti, A., Li, L., Alonso, J., Ecker, J., et al. (2006). Localization of iron in Arabidopsis seed requires the vacuolar membrane transporter VIT1. Science 314, 1295-1298. doi: 10.1126/science.1132563

Mary, V., Schnell Ramos, M., Gillet, C., Socha, A., Giraudat, J., Agorio, A., et al. (2015). Bypassing iron storage in endodermal vacuoles rescues the iron mobilization defect in the natural resistance associated-macrophage protein3natural resistance associated macrophage protein4 double mutant. Plant Physiol. 169, 748-759. doi: 10.1104/pp.15.00380

Murgia, I., Arosio, P., Tarantino, D., and Soave, C. (2012). Biofortification for combating 'hidden hunger' for iron. Trends Plant Sci. 17, 47-55. doi: 10.1016/j. tplants.2011.10.003

Ravet, K., and Pilon, M. (2013). Copper and iron homeostasis in plants: the challenges of oxidative stress. Antioxid. Redox. Signal. 19, 919-932. doi: 10. 1089/ars.2012.5084

Roschzttardtz, H., Conejero, G., Curie, C., and Mari, S. (2009). Identification of the endodermal vacuole as the iron storage compartment in the Arabidopsis embryo. Plant Physiol. 151, 1329-1338. doi: 10.1104/pp.109.144444

\section{ACKNOWLEDGMENTS}

The authors are greatly indebted to Xavier Jordana and María Isabel Gómez for their continued support and encouragement. We thank Nathalia Navarro for its technical support.

Roschzttardtz, H., Conejero, G., Curie, C., and Mari, S. (2010). Straightforward histochemical staining of Fe by the adaptation of an old-school technique: identification of the endodermal vacuole as the site of Fe storage in Arabidopsis embryos. Plant Signal. Behav. 5, 1-2. doi: 10.4161/psb.5.1.10159

Roschzttardtz, H., Paez-Valencia, J., Dittakavi, T., Jali, S., Reyes, F., Baisa, G., et al. (2014). The vasculature complexity and connectivity (VCC) gene encodes a plant-specific protein required for embryo provasculature development. Plant Physiol. 166, 889-902. doi: 10.1104/pp.114.246314

Scarpella, E., Barkoulas, M., and Tsiantis, M. (2010). Control of leaf and vein development by auxin. Cold Spring Harb. Protoc. 2:a001511. doi: 10.1101/ cshperspect.a001511

Sieburth, L., and Deyholos, M. (2006). Vascular development: the long and winding road. Curr. Opin. Plant Biol. 9, 48-54. doi: 10.1016/j.pbi.2005.11.008

Sieburth, L., Muday, G., King, E., Benton, G., Kim, S., Metcalf, K., et al. (2006). Scarface encodes an ARF-GAP that is required for normal auxin efflux and vein patterning in Arabidopsis. Plant Cell 18, 1396-1411. doi: 10.1105/tpc.105. 039008

Truernit, E., Bauby, H., Belcram, K., Barthélémy, J., and Palauqui, J. (2012). Octopus, a polarly localised membrane-associated protein, regulates phloem differentiation entry in Arabidopsis thaliana. Development 139, 1306-1315. doi: 10.1242/dev.072629

Vasconcelos, M., Gruissem, W., and Bhullar, N. (2016). Iron biofortification in the 21st century: setting realistic targets, overcoming obstacles, and new strategies for healthy nutrition. Curr. Opin. Biotechnol. 44, 8-15. doi: 10.1016/j.copbio. 2016.10.001

Waters, B., and Grusak, M. (2008). Quantitative trait locus mapping for seed mineral concentrations in two Arabidopsis thaliana recombinant inbred populations. New Phytol. 179, 1033-1047. doi: 10.1111/j.1469-8137.2008. 02544.x

Conflict of Interest Statement: The authors declare that the research was conducted in the absence of any commercial or financial relationships that could be construed as a potential conflict of interest.

Copyright (C) 2017 Roschzttardtz, Bustos, Coronas, Ibeas, Grant-Grant and VargasPérez. This is an open-access article distributed under the terms of the Creative Commons Attribution License (CC BY). The use, distribution or reproduction in other forums is permitted, provided the original author(s) or licensor are credited and that the original publication in this journal is cited, in accordance with accepted academic practice. No use, distribution or reproduction is permitted which does not comply with these terms. 